\title{
Prognostic value of MTA1, SOX4 and EZH2 expression in esophageal squamous cell carcinoma
}

\author{
WENYING LIU ${ }^{1 *}$, YU XIA ${ }^{2 *}$, MENGYAN LI ${ }^{1}$, GULINAER ABULAJIANG ${ }^{1}$, HUI WANG $^{1}$, LIPING SU $^{1}$, \\ $\mathrm{CHAO} \mathrm{LI}^{1}$, YAN SHI ${ }^{1}$, WENJING ZHANG ${ }^{1}$, SHANSHAN XU ${ }^{1}$ and YUQING MA ${ }^{1}$ \\ Departments of ${ }^{1}$ Pathology and ${ }^{2}$ Respiratory Medicine, The First Affiliated Hospital, \\ Xinjiang Medical University, Urumqi, Xinjiang 830054, P.R. China
}

Received September 9, 2020; Accepted March 10, 2021

DOI: $10.3892 /$ etm.2021.10154

\begin{abstract}
Esophageal cancer has always been one of the major malignant tumor types affecting the health of the Chinese population. Metastasis-associated protein 1 (MTA1), SOX4 and enhancer of zeste homolog $2(\mathrm{EZH} 2)$ are all potent inducers of invasion and metastasis in esophageal squamous cell carcinoma (ESCC). However, the role of these signaling molecules and their implication in ESCC have remained largely elusive. In the present study, the effects of MTA1, SOX 4 and EZH2 on the prognosis of patients with ESCC were explored. Immunohistochemistry was used to examine the expression levels of MTA1, SOX4 and EZH2. The $\chi^{2}$ test was used to analyze the association between protein expression and clinicopathological parameters. Kaplan-Meier curves and Cox proportional hazards model survival analysis was performed to investigate the effects of the three proteins examined on disease prognosis. The results indicated that MTA1 may be used as a prognostic and diagnostic marker for ESCC. To the best of our knowledge, the present study was the first to demonstrate that MTA1-SOX4 signaling is associated with prognosis in ESCC. However, no significant association was noted between SOX4 and EZH2 in the present study, which was inconsistent with previously reported findings. The function of the MTA1-SOX4-EZH2 axis and the interactions of the proteins involved require further investigation.
\end{abstract}

Correspondence to: Professor Yuqing Ma, Department of Pathology, The First Affiliated Hospital, Xinjiang Medical University, 393 Xinyi Road, Urumqi, Xinjiang 830054, P.R. China E-mail: yuqingm0928@126.com

*Contributed equally

Key words: esophageal squamous cell carcinoma, immunohistochemistry, prognosis, metastasis-associated protein 1, SOX4, enhancer of zeste homolog 2

\section{Introduction}

Esophageal squamous cell carcinoma (ESCC) is one of the most common types of malignant disease in the world and particularly in China (1). It is the fourth most common cause of cancer-associated death in China (1). Xinjiang is one of the areas with a high incidence of ESCC. Of note, in Kazakh, the mortality rate for ESCC is as high as $68.88 \%(2,3)$. Due to extensive lymphatic drainage, the majority of patients with ESCC are diagnosed at a late stage and the malignancy easily metastasizes at an early stage $(4,5)$. The treatment of ESCC is based on surgery combined with radiotherapy or chemotherapy $(4,5)$. The discovery of genes associated with the occurrence and development of the disease and investigation of the underlying mechanisms are of great significance for the development of specific targeted drugs that may be used for the treatment of this disease.

Our team has been investigating the classical Wnt signaling pathway as a molecular mechanism associated with the occurrence and development of ESCC (6). In cervical cancer, enhancer of zeste homolog $2(\mathrm{EZH} 2)$ promotes cell proliferation and tumor formation in cervical cancer through activation of the Wnt/ $\beta$-catenin pathway via GSK-3 $\beta$ - and TP53-mediated epigenetic silencing (7). However, the association of EZH2 with the development of ESCC and associated molecular mechanisms have remained largely elusive.

EZH2 is a key component of the polycomb repressive complex 2 complex, which catalyzes the trimethylation of histone $\mathrm{H} 3$ lysine 27 to promote transcriptional silencing that maintains cell integrity during development by promoting chromatin modifications $(7,8)$. EZH2 promotes cancer formation and progression through epigenetic activation of oncogenic signaling cascades and inhibition of pro-differentiation pathways (9). Studies have suggested that EZH2 is overexpressed in cancer and functions as an oncogene. Its expression correlates with poor patient prognosis in various cancer types by mediating the expression of target genes involved in tumorigenesis (10-12). In addition, EZH2 has been indicated to act as an epigenetic modifier during the TGF- $\beta$-induced epithelial-mesenchymal transition (EMT) (12). It was also reported that EZH2 is a member of the SRY-related HMG box (SOX) family signaling cascade and SOX4 directly activates EZH2 (13-15). 
SOX4 is the core protein involved in ESCC and its overexpression is attributed to both gene amplification and activation of the PI3K, Wnt and TGF- $\beta$ pathways (16). It has been identified as a master regulator of invasion and metastasis, acting upstream of several EMT inducers; EMT is a cellular biological process involved in the migration of primary cancer cells to secondary sites to cause metastasis $(13,14)$. SOX4 regulates several processes, such as the induction of cell survival, stemness, EMT, migration and metastasis (16). Studies including that by Tiwari et al (14) delineated a pathway wherein TGF- $\beta$ stimulates SOX4 expression, thereby reprogramming the epigenome to elicit metastasis of cancers (17). SOX4 overexpression is elevated in a wide variety of human cancer types and correlates with cancer progression and poor prognosis in prostate cancer (18), cutaneous melanoma (19) and breast cancer (20), suggesting the potential role of SOX4 in tumor progression. It has been previously reported that SOX4 activates EZH2 in a variety of cancers $(10,13,15)$. Above all, the effects of the SOX4-EZH2 signaling pathway were indicated to be able to predict the presence of metastasis and invasion of ESCC, as well as poor prognosis of affected patients (21).

Metastasis-associated protein 1 (MTA1) is a well-known oncogene that drives the metastasis of various cancer types (22). The metastasis-associated proteins are a family of co-regulators, which include MTA1, MTA2 and MTA3. MTA members are primarily involved in regulating target gene expression through the deacetylation of histones (23). The effects of MTA1 are attributed to the regulation of various cancer-promoting processes. These include the canonical Wnt $1 / \beta$-catenin signaling pathway, the stabilization of hypoxia-inducible factor- $1 \alpha$ and the regulation of invasion and metastasis via repression of adhesion molecules including E-cadherin (24-27). In ESCC, MTA1 has been reported to promote tumor metastasis and invasion (21). MTA1 is able to regulate the expression of EMT-associated factors in both normal and cancerous cells $(8,28)$ and predict cancer aggressiveness and adverse clinical outcomes in a wide range of tumor types, as evidenced by previous studies by our and other groups (29-31). It was predicted that the role of MTA1 in ESCC may be associated with SOX4. The purpose of the present study was to determine the expression levels of MTA1, SOX4 and EZH2 in ESCC and to investigate their association with clinicopathological parameters. The effects of MTA1, SOX4 and EZH2 on the prognosis of ESCC were analyzed in detail. The association between MTA1, SOX4 and EZH2 was analyzed by Spearman correlation analysis. The aim of the present study was to identify target genes that inhibit the proliferation of ESCC cells to facilitate the development of targeted drugs for the treatment of ESCC. Determination of the function of the MTA1/SOX4/EZH2 axis in ESCC and its influence on clinicopathological parameters and disease prognosis may lead to the development of strategies to predict and improve outcomes of ESCC.

\section{Materials and methods}

Patients and tissue samples. A total of 229 cases of ESCC were collected from January 2008 to December 2018 at the First Affiliated Hospital of Xinjiang Medical University (Urumqi, China). The inclusion criteria were as follows:
Patients diagnosed with ESCC between January 2008 and December 2018, the presence of SCC of the esophagus, the absence of radiotherapy or chemotherapy prior to surgery, esophageal malignancy as the major treatment focus and subjects of the Han and Kazakh ethnicities. The exclusion criteria were as follows: Adenocarcinoma of the esophagus, patients who had received radiotherapy or chemotherapy prior to surgery, tumor metastases to the esophagus and other ethnicities, such as Uygur and Mongolian. A total of 229 patients with ESCC were randomly selected for the present study. Paraffin-embedded ESCC tissues and matched noncancerous tissues were collected from a total of 229 cases, 119 of which were of Han and 110 of Kazak ethnicity. The resected specimens of these 229 patients were diagnosed as ESCCs at the pathology department. All 229 patients were treated by surgery and without any preoperative radiochemotherapy. The following information was recorded for each patient: Age, sex, ethnicity, tumor location, tumor size, degree of differentiation and TNM staging, lymph node status, vascular invasion, nerve invasion, postoperative radio-chemotherapy and progression of disease (32). The present study was approved by the Ethics committee of the First Affiliated Hospital of Xinjiang Medical University (Urumqi, China). The follow-up ended in July 2020 and information was obtained from the patients' medical records and telephone calls.

Tissue microarray (TMA) and immunohistochemistry (IHC). Paraffin-embedded tissue blocks of representative tumor and normal control tissue samples were selected by reviewing the hematoxylin and eosin-stained slides. Tissue cores with a diameter of $1.5 \mathrm{~mm}$ were extracted from each donor block and precisely arrayed into a new paraffin receptacle block with a maximum of 200 cores by using the Organization Microarrayer (Pathology Devices, Inc.). The sections (4 $\mu \mathrm{m})$ were obtained from formalin-fixed and paraffin-embedded TMA blocks, mounted on poly-L-lysine-coated glass slides and used for IHC.

The sections were deparaffinized with xylene, rehydrated in a graded alcohol series and heated in a microwave oven for antigen retrieval. To enhance antigen retrieval, the slides were autoclaved for $20 \mathrm{~min}$ in $1 \%$ sodium citrate buffer ( $\mathrm{pH} \mathrm{6.0)}$ and subsequently left at room temperature. Endogenous peroxidase activity was blocked with $3 \% \mathrm{H}_{2} \mathrm{O}_{2}$ at $37^{\circ} \mathrm{C}$ for $20 \mathrm{~min}$. The sections were incubated with primary antibodies against SOX4 (cat. no. bs-11208R; 1:200 dilution; BIOSS), MTA1 (cat. no. bs-1412R; 1:200 dilution; BIOSS) and EZH2 (cat. no. 5246; 1:50 dilution; Cell Signaling Technology, Inc.) overnight at $4^{\circ} \mathrm{C}$. After the primary antibodies were rinsed off, the sections were incubated with biotinylated secondary antibody (cat. no. SP-9001; OriGene Technologies, Inc.) for $30 \mathrm{~min}$ at $37^{\circ} \mathrm{C}$. The sections were then incubated with streptavidin horseradish peroxidase for an additional 30 min (LSAB kit; Dako; Agilent Technologies, Inc.) and stained with 3,3'-diaminobenzidine. The sections were counterstained with hematoxylin, dehydrated and mounted. Any sections in which primary antibodies were omitted were used as negative controls.

Evaluation of IHC. Immunostaining was examined under a light microscope by two pathologists who were blinded to the experimental conditions. The intensity of immunoreactivity 
was scored as follows: 0 , no staining; 1 , weak staining; 2 , moderate staining; and 3 , strong staining. The percentage of stained cells ranged from 0 to $100 \%$. The percentage of positive cells was scored for SOX4 expression according to the following criteria: 0 ( $0 \%$ positively stained tumor cells), 1 (1-25\% positively stained tumor cells), 2 (26-50\% positively stained tumor cells), 3 (51-75\% positively stained tumor cells) and 4 (76-100\% positively stained tumor cells). MTA1 was located in the cytoplasm and nucleus and its expression intensity was scored as follows: 0 (none), $1(<10 \%$ positively stained tumor cells), 2 (11-50\% positively stained tumor cells), 3 (51-80\% positively stained tumor cells) and 4 (81-100\% positively stained tumor cells). The staining index was calculated using the following formula: Percentage of positive cells $\mathrm{x}$ staining intensity score. The possible staining indexes calculated were $0,1,2,3,4,6,9$ and 12 . For MTA1 and SOX4, samples with a score of $\geq 4$ were considered positive. For EZH2 expression, tumor cells with nuclear staining were considered positive and all scores were applied to discriminate between positive (score $\geq 3$ ) and negative (score $<3$ ) staining $(18,33,34)$.

Statistical analysis. The association of expression data with clinicopathological characteristics was determined with the $\chi^{2}$ and the Fisher's exact tests. The association between the expression levels of MTA1, SOX4 and EZH2 was assessed by Spearman correlation analysis. As survival outcomes, overall survival (OS) and progression-free survival (PFS) were determined. PFS was defined as the time from diagnosis of ESCC to the time of tumor progression or death. Univariate analyses were used to assess the impact of various parameters on survival in the Kaplan-Meier; univariate and multivariate analyses were used to assess the impact of various parameters on survival in via Cox hazard regression analysis. The Kaplan-Meier method was used to analyze the clinicopathological parameters and tumor marker expression associated with the prognosis of ESCC. Cox hazard regression analysis was an additional analysis of independent factors associated with the prognosis of ESCC on the basis of the Kaplan-Meier method, log-rank tests were used to determine significant differences between Kaplan-Meier curves. The covariates of the Cox hazard regression analysis included MTA1, SOX4 and EZH2 expression, age, sex, ethnicity, tumor location, tumor size, degree of differentiation, TNM stage, lymph node status, vascular invasion, nerve invasion and postoperative radiochemotherapy. The results of the Cox hazard regression analysis and multinomial logistic regression analysis are expressed as the hazard ratio (HR) and 95\% CI. $\mathrm{P}<0.05$ was considered to indicate a statistically significant difference. All data were processed and statistically analyzed using SPSS statistics 23.0 software (IBM Corp.).

\section{Results}

Expression of MTA1, SOX4 and EZH2 and its association with clinicopathological parameters of ESCC. Clinicopathological data for the cohort are listed in Table SI. The results of the IHC staining demonstrated that EZH2 expression was localized in the nucleus of tumor cells. Two patterns of expression of MTA1 and SOX4 were evident, namely in the cytoplasm and nucleus (Fig. 1A-I). In normal esophageal tissues, MTA1, SOX4 and
EZH2 were not expressed or only expressed in the basal layer cells. Among the 229 cases of ESCC, 194 were positive for MTA1 expression and 35 cases were negative, resulting in a positive rate of MTA1 expression in ESCC of $84.72 \%$. A total of 152 cases were positive for SOX4, while 76 cases were negative (positive rate for SOX4, 66.81\%). EZH2 expression was positive in 95 and negative in 134 cases of ESCC (positive rate, $41.48 \%$ ). However, they were completely negative in normal control tissues. The association between the positive expression of the three proteins and the clinicopathological parameters was assessed in the 229 cases of ESCC. The association of expression data with clinicopathological characteristics was determined. High expression of MTA1 was associated with ethnicity $(\mathrm{P}<0.001)$ and lymph node metastasis $(\mathrm{P}=0.037)$; high expression of SOX2 was associated with age $(\mathrm{P}=0.016)$ and ethnicity $(\mathrm{P}=0.035)$; and high expression of EZH2 was associated with degree of differentiation $(\mathrm{P}=0.003)$. The results are presented in Table I.

Association between the expression levels of MTA1, SOX4 and $E Z H 2$. The correlations among the expression levels of MTA1, SOX4 and EZH2 determined by IHC staining were assessed in the 229 cases of ESCC. The expression of MTA1 was positively correlated with SOX4 expression, as determined using Spearman's correlation analysis $(\mathrm{Q}=0.139 ; \mathrm{P}=0.036$; Table II). The expression of MTA1 was not significantly correlated with that of EZH2 ( $\mathrm{Q}=0.087 ; \mathrm{P}=0.191$; Table III); he expression of SOX4 did not exhibit any correlation with that of EZH2 $(\mathrm{Q}=-0.122 ; \mathrm{P}=0.066$; Table II). This was different from the experimental results reported in other studies (14-15), possibly due to inter-individual differences, the sample size of the cohort and the ethnic groups Kazak that were included (Table III).

Prognostic factors for OS and PFS. The 3-year and 5-year survival rates were calculated separately in order to obtain more accurate data on prognosis. Kaplan-Meier and Cox hazard regression analysis were proposed to assess disease prognosis. The 5-year survival rate was $24.4 \%$ and the 3 -year survival rate was $37.1 \%$ for the 229 cases of ESCC.

Kaplan-Meier survival analysis indicated that MTA1 expression had an impact on the OS of patients with ESCC. Statistical analysis of 3-year survival demonstrated that it was significantly associated with MTA1 expression $\left(\chi^{2}=7.460\right.$; $\mathrm{P}=0.006$; Fig. 2A). Further subgroup analysis (Table IV) indicated that MTA1 expression also significantly affected survival in patients based on age ( $\geq 60$ group; $P=0.024)$, sex (male group; $\mathrm{P}=0.001$ ), ethnicity (Kazakh group; $\mathrm{P}=0.013$ ), tumor size ( $<3 \mathrm{~cm}$ group; $\mathrm{P}=0.031)$, degree of differentiation (moderate degree of differentiation group; $\mathrm{P}=0.014$ ), lymph node metastasis (no specific group; $\mathrm{P}=0.043$ ), depth of invasion (full-thickness group; $\mathrm{P}=0.006$ ), TNM stage (IVA+B group; $\mathrm{P}=0.043$ ), vascular invasion (no invasion group; $\mathrm{P}=0.034$ ), nerve invasion (no invasion group; $\mathrm{P}=0.006$ ), hematogenous metastasis (no metastasis group; $\mathrm{P}=0.009$ ) and in postoperative chemoradiotherapy (no chemotherapy group; $\mathrm{P}=0.031$ ). Statistical analysis of 5-year survival revealed a significant association of the latter with MTA1 expression levels in all groups $\left(\chi^{2}=4.198 ; P=0.040 ;\right.$ Fig. $\left.2 B\right)$. Further subgroup analysis indicated that MTA1 expression also significantly affected survival in patients of a specific sex 


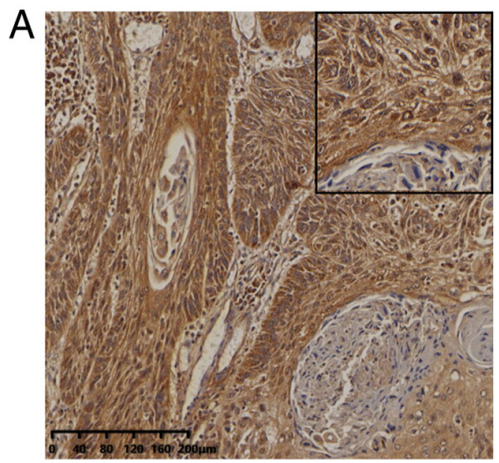

B

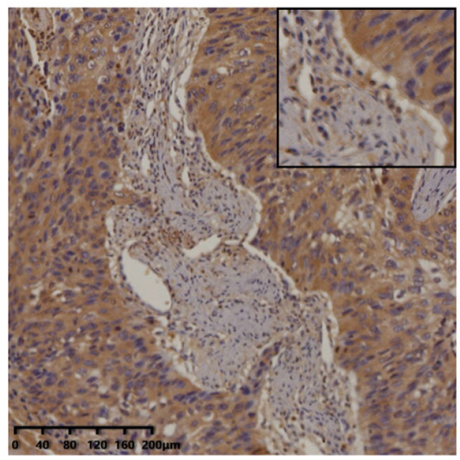

D

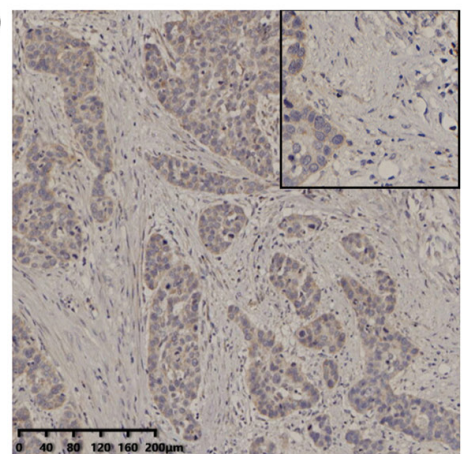

G

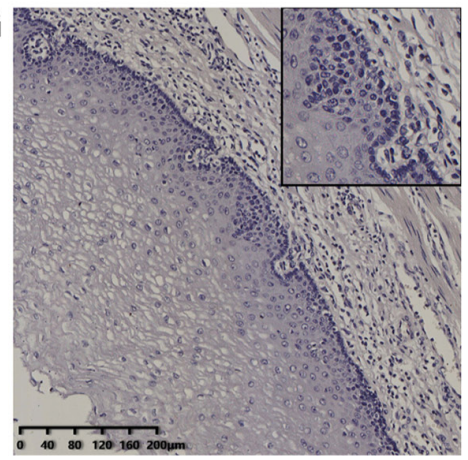

E

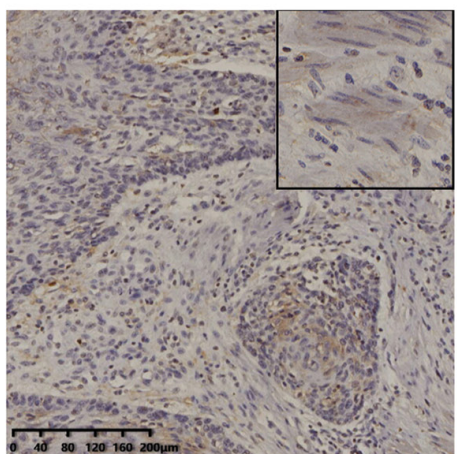

$\mathrm{H}$

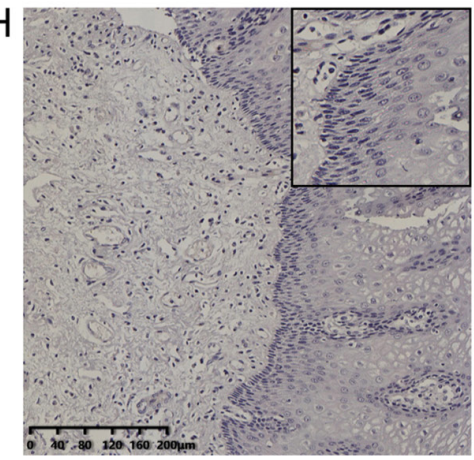

C

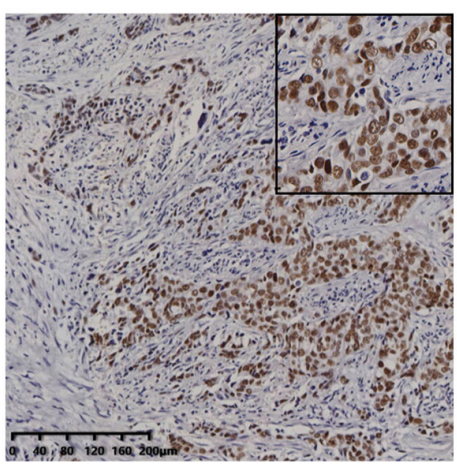

F
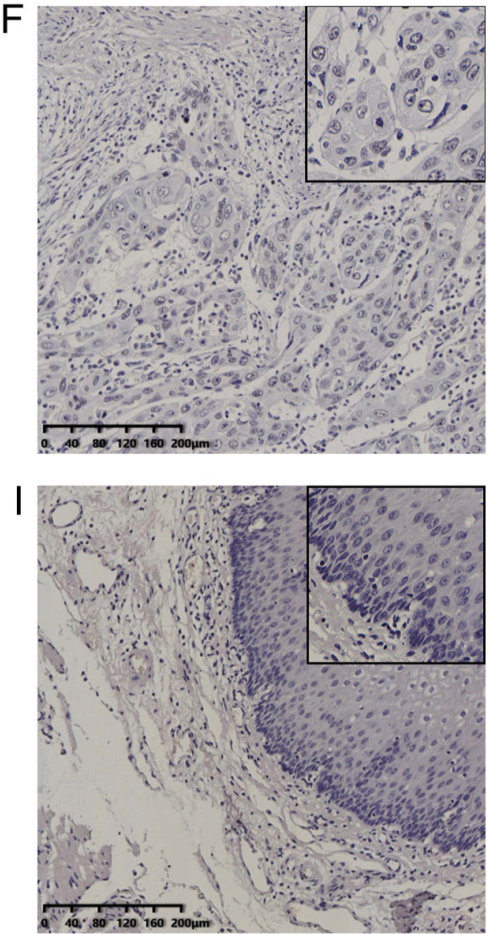

Figure 1. Expression of MTA1, SOX4 and EZH2 in ESCC and normal esophageal tissues. Immunohistochemical staining of ESCC for (A) MTA1 (positive), (B) SOX4 (positive), (C) EZH2 (positive), (D) SOX4 (negative), (E) SOX4 (negative) and (F) EZH2 (negative). Staining of normal esophageal tissues for (G and H) SOX4 (negative) and (I) EZH2 (negative) (magnification, x100 or x400 in magnified window; scale bar, $200 \mu \mathrm{m}$ ). ESCC, esophageal squamous cell carcinoma; MTA1, metastasis-associated protein 1; EZH2, enhancer of zeste homolog 2.

(male group; $\mathrm{P}=0.020$ ), ethnicity (Kazakh group; $\mathrm{P}=0.010$ ), depth of invasion (full-thickness group; $\mathrm{P}=0.029$ ) degree of differentiation (moderate degree of differentiation group; $\mathrm{P}=0.012$ ) and in nerve invasion (no invasion group; $\mathrm{P}=0.022$ ); $\chi^{2}$ analysis of the influence of MTA1 expression (positive vs. negative) on survival in the above subgroups is presented in Table IV. The use of the survival rate based on different clinicopathological parameters is more beneficial to accurately predict the prognosis and survival time of patients and the conclusions drawn are more reliable. By using this comparison, specific patients with different clinicopathological parameters were selected to target MTA1 and achieve precision therapy.

Furthermore, the HR was analyzed in the present study. By using Kaplan-Meier survival analysis, the OS at the follow-up date of patients with ESCC was assessed depending on MTA1 expression (positive vs. negative; $\chi^{2}=5.229 ; \mathrm{P}=0.022 ;$ Fig. $2 \mathrm{C}$ ). The degree of differentiation (PD vs. MD vs. WD; $\mathrm{P}=0.025$ ), TNM staging (I vs. II vs. III vs. IV; $\mathrm{P}=0.002$ ), lymph node metastasis (no vs. yes; $\mathrm{P}=0.003$ ) and nerve invasion (no vs. yes; $\mathrm{P}=0.010)$ also had a significant impact on $\mathrm{OS}$ at the follow-up date (Fig. 3A-C). Kaplan-Meier survival analysis and log-rank results are presented in Table V. However, further multivariate regression analysis indicated that only the degree of differentiation (HR: 0.765; 95\% CI: 0.607-0.965; $\mathrm{P}=0.023$ ), nerve invasion (HR: 1.451; 95\% CI: 1.012-2.079; $\mathrm{P}=0.043$ ) and MTA1 expression (HR: 1.565; 95\% CI: 0.355-0.899; $\mathrm{P}=0.016$ ) had an impact on OS (Table V). With regard to PFS, significant differences were noted between subjects with different tumor size ( $\geq 3 \mathrm{~cm}$ vs. $<3 \mathrm{~cm} ; \chi^{2}=4.432 ; \mathrm{P}=0.035$ ), degree of differentiation (PD vs. MD vs. WD; $\chi^{2}=12.282 ; \mathrm{P}=0.002$ ), TNM stage (I vs. II vs. III vs. IV; $\chi^{2}=15.805 ; \mathrm{P}=0.001$ ), lymph node metastasis (no vs. yes; $\chi^{2}=17.046 ; \mathrm{P}<0.001$ ), nerve invasion (no vs. yes; $\chi^{2}=4.938 ; \mathrm{P}=0.026$ ) and hematogenous metastasis (no vs. yes; $\chi^{2}=3.952 ; \mathrm{P}=0.047$ ), as determined by univariate analysis. However, multivariate analysis indicated that only the degree of differentiation (HR: 0.723 ; 95\% CI: $0.577-0.907$; $\mathrm{P}=0.005$ ) and nerve invasion (HR: 1.465; 95\% CI: 1.029-2.087; $\mathrm{P}=0.034$ ) had an independent impact on PFS (Table V). These 
Table I. Association between MTA1, SOX4 and EZH2 expression and clinicopathological parameters in esophageal squamous cell carcinoma.

\begin{tabular}{|c|c|c|c|c|c|c|c|c|c|}
\hline \multirow[b]{2}{*}{ Item } & \multicolumn{3}{|c|}{ MTA1 } & \multicolumn{3}{|c|}{ SOX4 } & \multicolumn{3}{|c|}{ EZH2 } \\
\hline & Negative & Positive & P-value & Negative & Positive & P-value & Negative & Positive & P-value \\
\hline Age (years) & & & 0.557 & & & 0.016 & & & 0.092 \\
\hline$<60$ & $11(4.8)$ & $71(31.0)$ & & $19(8.3)$ & $63(27.5)$ & & $54(23.6)$ & $28(12.2)$ & \\
\hline$\geq 60$ & $24(10.5)$ & $123(53.7)$ & & $57(24.9)$ & $90(39.3)$ & & $80(34.9)$ & $67(29.3)$ & \\
\hline Ethnicity & & & $<0.001$ & & & 0.035 & & & 0.130 \\
\hline Han & $7(3.1)$ & 112 (48.9) & & $32(14.0)$ & $87(38.0)$ & & $64(27.9)$ & $55(24.0)$ & \\
\hline Kazakh & $28(12.2)$ & $82(35.8)$ & & 44 (19.2) & $66(28.8)$ & & $70(30.6)$ & $40(17.5)$ & \\
\hline $\begin{array}{l}\text { Degree of } \\
\text { differentiation }\end{array}$ & & & 0.395 & & & 0.725 & & & 0.003 \\
\hline PD & $10(4.4)$ & $36(15.7)$ & & $13(5.7)$ & $33(14.4)$ & & $35(15.3)$ & $11(4.8)$ & \\
\hline MD & $17(7.4)$ & $106(46.3)$ & & $42(18.3)$ & $81(35.4)$ & & 73 (31.9) & $50(21.8)$ & \\
\hline WD & $8(3.5)$ & $52(22.7)$ & & $21(9.2)$ & $39(17.0)$ & & $26(11.4)$ & $34(14.8)$ & \\
\hline $\begin{array}{l}\text { Lymph node } \\
\text { metastasis }\end{array}$ & & & 0.037 & & & 0.665 & & & 0.289 \\
\hline No & $29(12.7)$ & $126(55.0)$ & & $50(21.8)$ & 105 (45.9) & & $87(38.0)$ & $68(29.7)$ & \\
\hline Yes & $6(2.6)$ & $68(29.7)$ & & $26(11.4)$ & $48(21.0)$ & & $47(20.5)$ & $27(11.8)$ & \\
\hline
\end{tabular}

Values are expressed as n (\%). MTA1, metastasis-associated protein 1; EZH2, enhancer of zeste homolog 2; PD, poorly differentiated; $\mathrm{MD}$, moderately differentiated; $\mathrm{WD}$, well differentiated.

Table II. Correlation of SOX4 with MTA1 and EZH2.

\begin{tabular}{lrrrr}
\hline & \multicolumn{3}{c}{ SOX4 } & \\
\cline { 2 - 3 } Item & - & + & $\varrho$ & P-value \\
\hline MTA1 & & & 0.139 & 0.036 \\
- & $17(7.4)$ & $18(7.9)$ & & \\
+ & $59(25.8)$ & $135(59.0)$ & & \\
EZH2 & & & -0.122 & 0.066 \\
- & $38(16.6)$ & $96(41.9)$ & & \\
+ & $38(16.6)$ & $57(24.9)$ & & \\
\hline
\end{tabular}

Values are expressed as n (\%). MTA1, metastasis-associated protein 1; EZH2, enhancer of zeste homolog 2.

data provided ample evidence that MTA1 may be used as an independent prognostic and diagnostic marker for ESCC and for the prediction of survival of patients with ESCC.

\section{Discussion}

In the present study, the expression levels of MTA1, SOX4 and $\mathrm{EZH} 2$ were investigated in clinical specimens and the association between the expression of these markers and several histopathological factors related to clinical outcome was assessed. Cancer metastasis represents a major challenge in ESCC treatment. The change in the expression levels of certain factors is a characteristic feature of ESCC
Table III. Correlation of MTA1 with EZH2.

\begin{tabular}{lrrrr}
\hline & \multicolumn{2}{c}{ MTA1 } & & \\
\cline { 2 - 3 } Item & - & + & 0 & P-value \\
\hline EZH2 & & & 0.087 & 0.191 \\
- & $24(10.5)$ & $110(48.0)$ & & \\
+ & $11(48.0)$ & $84(36.7)$ & & \\
\hline
\end{tabular}

Values are expressed as n (\%). MTA1, metastasis-associated protein 1; $\mathrm{EZH} 2$, enhancer of zeste homolog 2 .

and is a prerequisite for tumor metastasis. Therefore, a clear understanding of the molecular mechanisms underlying the progression of ESCC is of pivotal importance for the prevention of its metastasis. MTA1 is a constitutive component of the NuRD complex and is able to regulate gene transcription in both NuRD-dependent and NuRD-independent manners (33). MTA1 overexpression has been reported in various cancer types and leads to metastasis and poor prognosis (35). The mechanism of the role of MTA1 in ESCC metastasis remains elusive. A previous study analyzed data from the ESCC survival database and the results indicated that patients with ESCC, high MTA1 expression exhibited a significant association with tumor metastasis and poor prognosis (36). It has also been indicated that MTA1 is associated with tumor recurrence and metastasis in cervical and prostate cancers $(26,27)$. The present study demonstrated that the expression levels of MTA1 were significantly associated with prognosis of 

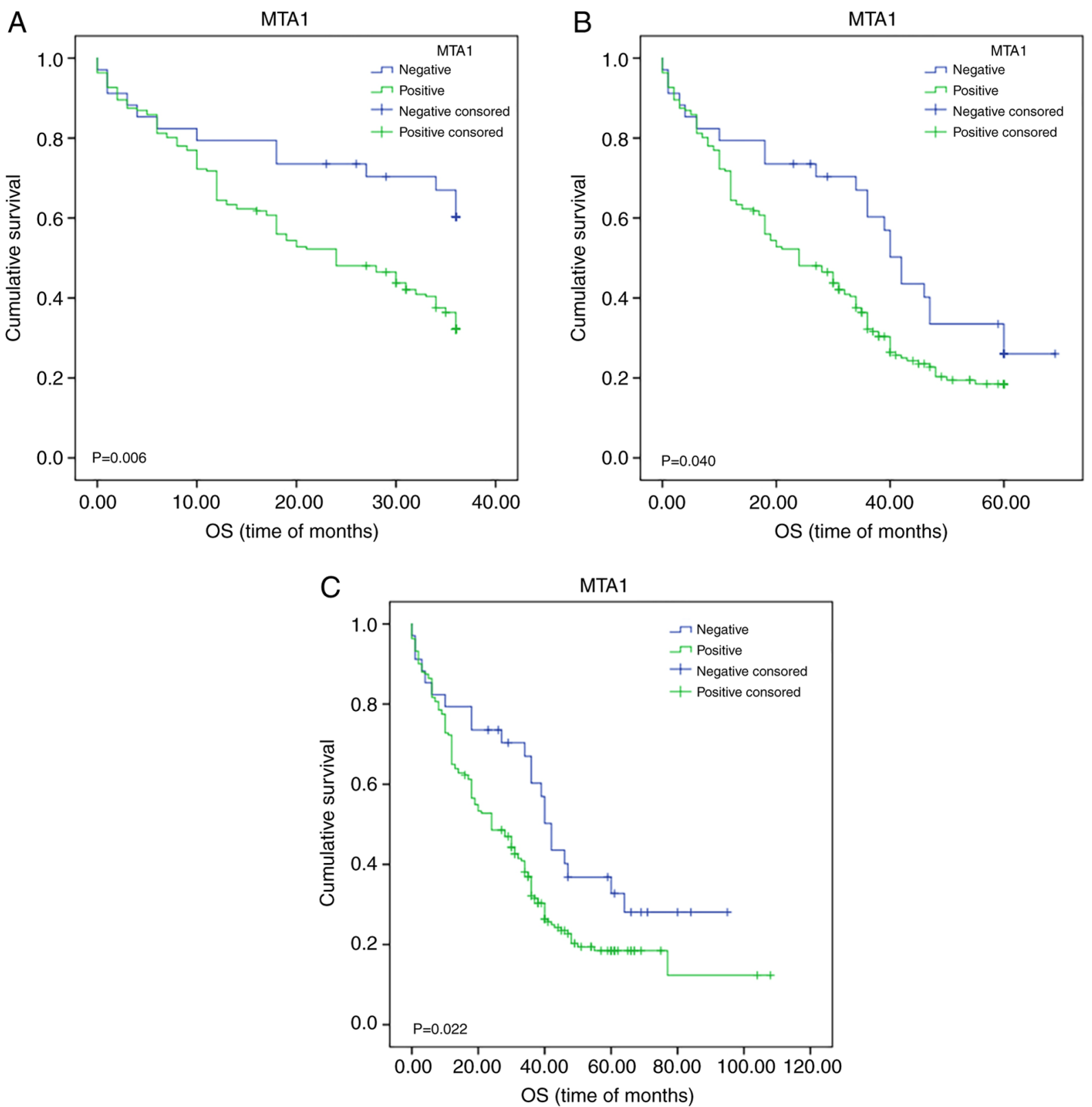

Figure 2. Kaplan-Meier survival analysis for patients with ESCC. (A) OS over three years; patients with ESCC and MTA1-positive status had poor OS. (B) OS over five years; patients with ESCC and MTA1-positive status had poor OS. (C) OS at the follow-up date; high expression of MTA1 in ESCC was associated with poor OS. OS, overall survival; ESCC, esophageal squamous cell carcinoma; MTA1, metastasis-associated protein 1.

patients with ESCC, which is consistent with previously reported results (21).

Furthermore, it was indicated that the MTA1-SOX4 axis is associated with ESCC progression. In the present study, the correlation between MTA1 and SOX4 expression was assessed in 229 ESCC tissues. In addition, the association with clinicopathological parameters and poor prognosis was assessed. Li et al (37) performed an expression profile analysis and verified the role of the MTA1-SOX4 regulatory axis in three different cancer cell lines. The migratory and invasive abilities of cancer cells overexpressing MTA1 were also assessed (37). Widespread co-expression of MTA1 and SOX4 has been determined in various cancer types using The Cancer Genome Atlas database. To the best of our knowledge, the present study was the first to demonstrate MTA1-SOX4 signaling in ESCC. Targeting a single factor provides less data than targeting a specific pathway.
Therefore, the discovery of the MTA1-SOX4 signaling pathway may provide potential treatment options for ESCC.

SOX4 has been associated with metastasis and poor prognosis and is induced by TGF- $\beta$. SOX 4 acts upstream of the EMT in order to promote invasion and metastasis $(13,19)$. Its mechanism is independent of the canonical TGF- $\beta$ signaling effector SMAD4, since short hairpin RNA-mediated ablation of SMAD4 did not substantially affect SOX4 expression in NMuMG cells (16). Li et al (37) demonstrated that both SOX4 and MTA1 were downstream effectors of TGF- $\beta$, whereas the deletion of either one substantially impaired the ability of TGF- $\beta$ to induce metastasis and invasion. MTA1 overexpression alone was sufficient to induce metastasis and invasion by activating SOX4 in the absence of TGF- $\beta$ (37). This also suggests that other factors leading to MTA1 upregulation in cancer may contribute 
Table IV. Association of MTA1 expression (positive vs. negative) with 3-year and 5-year OS in patient subgroups.

\begin{tabular}{|c|c|c|c|c|c|c|}
\hline \multirow[b]{2}{*}{ Parameter } & \multicolumn{2}{|c|}{ 3-year survival rate } & \multicolumn{2}{|c|}{ 5-year survival rate } & \multicolumn{2}{|c|}{ OS (at follow-up date) } \\
\hline & $\chi^{2}$ & P-value & $\chi^{2}$ & P-value & $\chi^{2}$ & P-value \\
\hline \multicolumn{7}{|c|}{ Age (years) } \\
\hline$<60$ & 2.196 & 0.138 & 3.325 & 0.068 & 3.325 & 0.068 \\
\hline$\geq 60$ & 5.062 & 0.024 & 1.314 & 0.252 & 1.922 & 0.166 \\
\hline \multicolumn{7}{|l|}{ Sex } \\
\hline Male & 11.981 & 0.001 & 5.435 & 0.020 & 6.685 & 0.010 \\
\hline Female & 0.269 & 0.604 & 0.001 & 0.980 & 0.001 & 0.980 \\
\hline \multicolumn{7}{|l|}{ Ethnicity } \\
\hline Han & 3.094 & 0.079 & 0.512 & 0.474 & 1.289 & 0.256 \\
\hline Kazakh & 6.199 & 0.013 & 6.632 & 0.010 & 6.541 & 0.011 \\
\hline \multicolumn{7}{|c|}{ Tumor location } \\
\hline Up & 1.525 & 0.217 & 1.525 & 1.525 & 1.525 & 0.217 \\
\hline M & 3.851 & 0.050 & 2.541 & 2.541 & 2.917 & 0.088 \\
\hline $\mathrm{L}$ & 2.361 & 0.124 & 0.666 & 0.666 & 1.115 & 0.291 \\
\hline \multicolumn{7}{|c|}{ Tumor size (cm) } \\
\hline$<3$ & 4.664 & 0.031 & 1.545 & 0.214 & 2.361 & 0.124 \\
\hline$\geq 3$ & 3.233 & 0.072 & 2.589 & 0.108 & 2.748 & 0.097 \\
\hline \multicolumn{7}{|c|}{ Degree of differentiation } \\
\hline PD & 3.724 & 0.054 & 1.377 & 0.241 & 2.027 & 0.155 \\
\hline MD & 6.064 & 0.014 & 6.366 & 0.012 & 6.663 & 0.010 \\
\hline WD & 0.342 & 0.559 & 0.001 & 0.976 & 0.009 & 0.923 \\
\hline \multicolumn{7}{|c|}{ Lymph node metastasis } \\
\hline No & 4.100 & 0.043 & 2.591 & 0.107 & 3.103 & 0.078 \\
\hline Yes & 1.655 & 0.198 & 0.552 & 0.457 & 0.506 & 0.477 \\
\hline \multicolumn{7}{|c|}{ Depth of invasion } \\
\hline MA & 0.616 & 0.432 & 0.616 & 0.432 & 0.616 & 0.432 \\
\hline MS & 0.588 & 0.443 & 0.183 & 0.669 & 0.546 & 0.460 \\
\hline FT & 7.691 & 0.006 & 4.760 & 0.029 & 5.066 & 0.024 \\
\hline \multicolumn{7}{|l|}{ TNM stage } \\
\hline $\mathrm{IA}+\mathrm{B}$ & 0.863 & 0.353 & 0.863 & 0.353 & 0.732 & 0.392 \\
\hline $\mathrm{IIA}+\mathrm{B}$ & 2.085 & 0.149 & 1.238 & 0.266 & 1.751 & 0.186 \\
\hline IIIA+B & 1.782 & 0.182 & 0.882 & 0.348 & 0.882 & 0.348 \\
\hline $\mathrm{IVA}+\mathrm{B}$ & 4.101 & 0.043 & 2.482 & 0.115 & 2.482 & 0.115 \\
\hline \multicolumn{7}{|c|}{ Vascular invasion } \\
\hline No & 4.470 & 0.034 & 2.553 & 0.110 & 2.999 & 0.083 \\
\hline Yes & 3.328 & 0.068 & 2.746 & 0.098 & 3.648 & 0.056 \\
\hline \multicolumn{7}{|c|}{ Nerve invasion } \\
\hline No & 7.591 & 0.006 & 5.215 & 0.022 & 6.476 & 0.011 \\
\hline Yes & 0.371 & 0.542 & 0.012 & 0.913 & 0.012 & 0.913 \\
\hline \multicolumn{7}{|c|}{ Hematogenous metastasis } \\
\hline No & 4.666 & 0.031 & 1.720 & 0.190 & 2.404 & 0.121 \\
\hline Yes & 3.164 & 0.075 & 2.869 & 0.090 & 2.893 & 0.089 \\
\hline
\end{tabular}

MTA1, metastasis-associated protein 1; OS, overall survival; Up, upper; M, middle; L, lower; MA, mucosa; MS, muscularis; FT, full thickness; $\mathrm{PD}$, poorly differentiated; MD, moderately differentiated; WD, well differentiated.

to metastasis and invasion by activating the MTA1-SOX4 signaling pathway. The present data demonstrated a significant correlation between the expression of SOX4 and MTA1, which also suggested that MTA1 may be used 

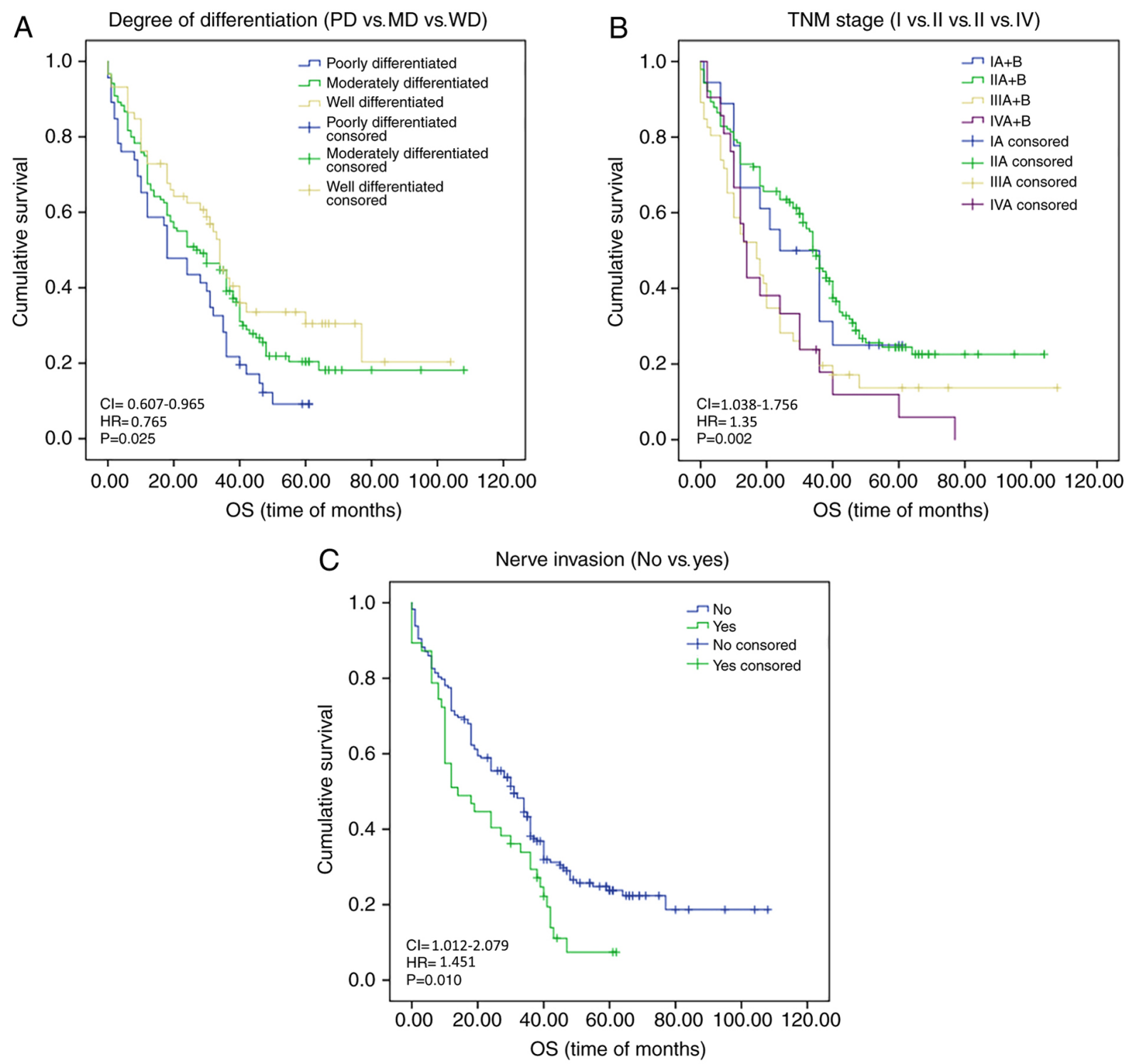

Figure 3. Kaplan-Meier survival analysis for OS (months). (A) Effect of degree of differentiation on OS in patients with ESCC. (B) Effect of TNM stage on OS in patients with ESCC. (C) Effect of nerve invasion status on OS in patients with ESCC. OS, overall survival; ESCC, esophageal squamous cell carcinoma; PD, poorly differentiated; MD, moderately differentiated; WD, well differentiated; HR, hazard ratio.

as a prognostic and diagnostic marker of ESCC, which was consistent with previous findings (37). In the present study, the data indicated that the expression status of SOX4 was closely associated with age $(\mathrm{P}=0.016)$ and ethnicity $(\mathrm{P}=0.035)$. However, SOX4 expression was not significantly associated with sex, tumor size, tumor location, degree of differentiation, AJCC stage, lymph node metastasis, vascular invasion, nerve invasion and hematogenous metastasis in ESCC. Age and ethnicity were assessed in the present analysis and the results suggested that the expression levels of MTA1 were significantly different between subjects with different sexes and among subjects with different ethnicity, which may aid in determining suitable recipients of different targeted therapies.

Previous studies have indicated that EZH2 is one of the direct transcriptional targets of SOX4 and that ablation of EZH2 function affects SOX4 expression (13). In addition, SOX4 is able to enhance the expression of EZH2 via binding to the promoter region (13), whereas it may also interact with EZH2 and histone deacetylase 3 in order to form a corepressor complex, which silences microRNA expression and affects metastasis and invasion (20). Studies have suggested that increased expression or activity of SOX4-EZH2 is a marker of advanced and metastatic disease in various solid tumor types and the SOX4-EZH2 axis is closely associated with disease progression in ovarian and pancreatic cancers $(13,20)$. Li et al $(37)$ demonstrated that EZH2 is a downstream target of the TGF- $\beta$-MTA1-SOX4 signaling axis in human small cell lung cancer, colorectal carcinoma and ovarian cancer. In the present study, the MTA1-positive group exhibited poor prognosis based on the OS rates. However, this does not affect the interpretation of the results, since SOX4 affects metastasis and invasion by regulating the expression of $\mathrm{EZH} 2$ in ESCCs, which has been previously reported (20). However, this was not confirmed by the results of the present study, which is inconsistent with 
Table V. Univariate and multivariate analysis of the influence of variables on OS and PFS.

\begin{tabular}{|c|c|c|c|c|c|c|c|c|}
\hline \multirow[b]{3}{*}{ Variable } & \multicolumn{4}{|c|}{ OS } & \multicolumn{4}{|c|}{ PFS } \\
\hline & \multicolumn{2}{|c|}{ Univariate } & \multicolumn{2}{|c|}{ Multivariate } & \multicolumn{2}{|c|}{ Univariate } & \multicolumn{2}{|c|}{ Multivariate } \\
\hline & $\chi^{2}$ & P-value & $\mathrm{HR}(95 \% \mathrm{CI})$ & P-value & $\chi^{2}$ & P-value & HR $(95 \% \mathrm{CI})$ & P-value \\
\hline Sex (female vs. male) & 2.146 & 0.143 & - & - & 1.762 & 0.184 & - & - \\
\hline Age ( $\geq 60$ years vs. $<60$ years) & 0.679 & 0.410 & - & - & 0.247 & 0.619 & - & - \\
\hline Ethnicity (Han vs. Kazakh) & 0.384 & 0.535 & - & - & 0.015 & 0.903 & - & - \\
\hline $\begin{array}{l}\text { Tumor location } \\
\text { (Up vs. M vs. L) }\end{array}$ & 0.271 & 0.873 & - & - & 0.771 & 0.680 & - & - \\
\hline Tumor size ( $\geq 3 \mathrm{~cm}$ vs. $<3 \mathrm{~cm})$ & 3.826 & 0.050 & - & - & 4.432 & 0.035 & $\begin{array}{c}1.327 \\
(0.942-1.870)\end{array}$ & 0.106 \\
\hline $\begin{array}{l}\text { Degree of differentiation } \\
\text { (PD vs. MD vs. WD) }\end{array}$ & 7.398 & 0.025 & $\begin{array}{c}\text { PD } 0.245 \\
(0.072-0.841)\end{array}$ & 0.025 & 12.282 & 0.002 & $\begin{array}{c}\text { PD } 0.106 \\
(0.020-0.548)\end{array}$ & 0.007 \\
\hline $\begin{array}{l}\text { Depth of invasion } \\
\text { (MA vs. MS vs. FT) }\end{array}$ & 2.400 & 0.301 & - & - & 3.346 & 0.188 & - & - \\
\hline $\begin{array}{l}\text { TNM stage } \\
\text { (I vs. II vs. III vs. IV) }\end{array}$ & 15.350 & 0.002 & - & - & 15.805 & 0.001 & - & - \\
\hline $\begin{array}{l}\text { Lymph node metastasis } \\
\text { (yes vs. no) }\end{array}$ & 8.707 & 0.003 & $\begin{array}{c}1.085 \\
(0.716-1.642)\end{array}$ & 0.701 & 17.046 & $<0.001$ & $\begin{array}{c}1.479 \\
(0.968-2.259)\end{array}$ & 0.071 \\
\hline Vascular invasion (yes vs. no) & 0.924 & 0.337 & - & - & 1.187 & 0.276 & - & - \\
\hline $\begin{array}{l}\text { Nerve invasion } \\
\text { (yes vs. no) }\end{array}$ & 6.720 & 0.010 & $\begin{array}{c}1.451 \\
(1.012-2.079)\end{array}$ & 0.043 & 4.938 & 0.026 & $\begin{array}{c}1.465 \\
(1.029-2.087)\end{array}$ & 0.034 \\
\hline $\begin{array}{l}\text { Postradiochemotherapy } \\
\text { (yes vs.no) }\end{array}$ & 0.003 & 0.953 & - & - & 0.224 & 0.636 & - & - \\
\hline $\begin{array}{l}\text { Hematogenous metastasis } \\
\text { (yes vs.no) }\end{array}$ & 1.425 & 0.433 & - & - & 3.952 & 0.047 & $\begin{array}{c}1.485 \\
(0.995-2.217)\end{array}$ & 0.053 \\
\hline $\begin{array}{l}\text { MTA1 expression } \\
\text { (positive vs. negative) }\end{array}$ & 5.229 & 0.022 & $\begin{array}{c}1.565 \\
(0.355-0.899)\end{array}$ & 0.016 & 2.004 & 0.157 & - & - \\
\hline $\begin{array}{l}\text { SOX4 expression } \\
\text { (positive vs. negative) }\end{array}$ & 0.013 & 0.908 & - & - & 0.006 & 0.941 & - & - \\
\hline $\begin{array}{l}\text { EZH2 expression } \\
\text { (positive vs. negative) }\end{array}$ & 0.807 & 0.369 & - & - & 0.657 & 0.481 & - & - \\
\hline
\end{tabular}

MTA1, metastasis-associated protein 1; EZH2, enhancer of zeste homolog 2; HR, hazard ratio; PD, poorly differentiated; MD, moderately differentiated; WD, well differentiated; OS, overall survival; PFS, progression-free survival; up, upper; M, middle; L, lower; MA, mucosa; MS, muscularis; FT, full thickness.

previous conclusions. Therefore, the clinical significance of SOX4-EZH2 requires further verification. In ESCC, the MTA1-SOX4 interaction may be associated with the improvement of clinical outcomes (37). In summary, the data of the present study validated MTA1 as a predictive marker for poor OS and PFS in patients with metastatic ESCC. In conclusion, the MTA1-SOX4 axis was associated with ESCC progression and MTA1 was identified as a novel, independent prognostic factor in ESCC. The results of the present study contradicted those of previous studies. The results may be different from those reported by other studies due to the small sample size and confounding bias. MTA1-SOX4 may serve as a key cascade in ESCC, which is important for metastasis, invasion and prognosis. In conclusion, high expression of MTA1 was associated with ethnicity and lymph node metastasis, whereas high expression of SOX4 was closely associated with age and ethnicity and high expression of EZH2 with the degree of differentiation. A positive correlation between MTA1 and SOX4 was identified. The results of univariate and multivariate analyses indicated that these two markers had an effect on prognosis. Therefore, MTA1 may be used as a molecular marker for screening for ESCC and prognostication of patients. It may be concluded that the activation of the MTA1-SOX4 axis may have a role in the development of ESCC and is associated with poor prognosis. The identification of targeted drugs that are able to inhibit MTA1 expression may provide a potential strategy to prolong the survival of patients with ESCC.

The present study was the first to propose the application of MTA1 expression as a marker in ESCC. Further studies are required to confirm these findings and facilitate the clinical application of MTA1 in ESCC. 


\section{Acknowledgements}

Not applicable.

\section{Funding}

This study was supported by grants from The Natural Science Foundation of Xinjiang Uygur Autonomous Region (grant no. 2018D01C182), The National Natural Science Foundation of China (grant no. 81860422), State Key Laboratory of Pathogenesis, Prevention and Treatment of High Incidence Diseases in Central Asia Fund (grant no. SKL-HIDCA-2020-4) and Xinjiang Medical University Clinical Medicine Peak Discipline supporting funds within the school (grant no. 33-0104006020801" ).

\section{Availability of data and materials}

The datasets used and/or analyzed during the current study are available from the corresponding author on reasonable request.

\section{Authors' contributions}

WYL, YX and YQM conceived and designed the study; GLA, MYL, HW and LPS performed the experiments; CL, YS, WJZ and SSX analyzed the data; WZ and ZM interpreted the data. WYL wrote the manuscript; WYL, YX and YQM gave final approval of the version to be published. All authors read and approved the final manuscript. All authors have checked and confirmed the authenticity of the raw data.

\section{Ethics approval and consent to participate}

The study protocol was approved by the Ethics Review Board of The First Affiliated Hospital of Xinjiang Medical University (approval no. 20180223-08). Written informed consent was obtained from all participants. All of the procedures were performed in accordance with the Declaration of Helsinki and relevant policies in China.

\section{Patient consent for publication}

Not applicable.

\section{Competing interests}

The authors declare that they have no competing interests.

\section{References}

1. Lin DC, Hao JJ, Nagata Y, Xu L, Shang L, Meng X, Sato Y, Okuno Y, Varela AM, Ding LW, et al: Genomic and molecular characterization of esophageal squamous cell carcinoma. Nat Genet 46: 467-473, 2014.

2. Jain R, Gupta S, Pasricha N, Faujdar M, Sharma M and Mishra P: ESCC with metastasis in the young age of caustic ingestion of shortest duration. J Gastrointest Cancer 41: 93-95, 2010.

3. Liu Q, Liang M, Liu T, Vuitton L, Zheng S, Gao X, Lu M, Li X, Sheyhidin I and Lu X: M2 isoform of pyruvate kinase (PKM2) is upregulated in Kazakh's ESCC and promotes proliferation and migration of ESCC cells. Tumour Biol 37: 2665-2672, 2016.
4. Encinas de la Iglesia J, Corral de la Calle MA,FernandezPerezGC, Ruano Perez R and Alvarez Delgado A: Esophageal cancer: Anatomic particularities, staging, and imaging techniques. Radiologia 58: 352-365, 2016.

5. Liu B, Bo Y, Wang K, Liu Y, Tang X, Zhao Y, Zhao E and Yuan L: Concurrent neoadjuvant chemoradiotherapy could improve survival outcomes for patients with esophageal cancer: A meta-analysis based on random clinical trials. Oncotarget 8: 20410-20417, 2017.

6. Zhou YX, Liu Q, Wang H, Ding F and Ma YQ: The expression and prognostic value of SOX2, $\beta$-catenin and survivin in esophageal squamous cell carcinoma. Future Oncol 15: 4181-4195, 2019.

7. Chen Q, Zheng PS and Yang WT: EZH2-mediated repression of GSK-3 $\beta$ and TP53 promotes Wnt $/ \beta$-catenin signaling-dependent cell expansion in cervical carcinoma. Oncotarget 7: 36115-36129, 2016.

8. Moon HE, Cheon H, Chun KH, Lee SK, Kim YS, Jung BK, Park JA, Kim SH, Jeong JW and Lee MS: Metastasis-associated protein 1 enhances angiogenesis by stabilization of HIF-1alpha. Oncol Rep 16: 929-935, 2006.

9. Ezhkova E, Pasolli HA, Parker JS, Stokes N, Su IH, Hannon G, Tarakhovsky A and Fuchs E: Ezh2 orchestrates gene expression for the stepwise differentiation of tissue-specific stem cells. Cell 136: 1122-1135, 2009.

10. Su IH, Basavaraj A, Krutchinsky AN, Hobert O, Ullrich A, Chait BT and Tarakhovsky A: Ezh2 controls B cell development through histone $\mathrm{H} 3$ methylation and Igh rearrangement. Nat Immunol 4: 124-131, 2003.

11. Nienstedt JC, Schroeder C, Clauditz T, Simon R, Sauter G, Muenscher A, Blessmann M, Hanken H and Pflug C: EZH2 overexpression in head and neck cancer is related to lymph node metastasis. J Oral Pathol Med 47: 240-245, 2018.

12. Feng H, Yu Z, Tian Y, Lee YY, Li MS, Go MY, Cheung YS, Lai PB, Chan AM, To KF, et al: A CCRK-EZH2 epigenetic circuitry drives hepatocarcinogenesis and associates with tumor recurrence and poor survival of patients. J Hepatol 62: 1100-1111, 2015.

13. Parvani JG and Schiemann WP: Sox4, EMT programs, and the metastatic progression of breast cancers: Mastering the masters of EMT. Breast Cancer Res 15: R72, 2013.

14. Tiwari N, Tiwari VK, Waldmeier L, Balwierz PJ, Arnold P, Pachkov M, Meyer-Schaller N, Schubeler D, van Nimwegen E and Christofori G: Sox 4 is a master regulator of epithelial-mesenchymal transition by controlling Ezh2 expression and epigenetic reprogramming. Cancer Cell 23: 768-783, 2013.

15. Lin L, Wang Z, Jin H, Shi H, Lu Z and Qi Z: miR-212/132 is epigenetically downregulated by SOX4/EZH2-H3K27me3 feedback loop in ovarian cancer cells. Biol: Nov 3, 2016 (Epub ahead of print).

16. Moreno CS: SOX4: The unappreciated oncogene. Semin Cancer Biol 67: 57-64, 2020.

17. David CJ, Huang YH, Chen M, Su J, Zou Y, Bardeesy N, Iacobuzio-Donahue CA and Massague J: TGF- $\beta$ tumor suppression through a lethal EMT. Cell 164: 1015-1030, 2016.

18. Wang L, Zhang J, Yang X, Chang YW, Qi M, Zhou Z, Zhang J and Han B: SOX4 is associated with poor prognosis in prostate cancer and promotes epithelial-mesenchymal transition in vitro. Prostate Cancer Prostatic Dis 16: 301-307, 2013.

19. Jafarnejad SM, Wani AA, Martinka M and Li G: Prognostic significance of Sox 4 expression in human cutaneous melanoma and its role in cell migration and invasion. Am J Pathol 177: 2741-2752, 2010 .

20. Zhang J, Liang Q, Lei Y, Yao M, Li L, Gao X, Feng J, Zhang Y, Gao H, Liu DX, et al: SOX4 induces epithelial-mesenchymal transition and contributes to breast cancer progression. Cancer Res 72: 4597-4608, 2012.

21. Koumangoye RB, Andl T, Taubenslag KJ, Zilberman ST, Taylor CJ, Loomans HA and Andl CD: SOX4 interacts with EZH2 and HDAC3 to suppress microRNA-31 in invasive esophageal cancer cells. Mol Cancer 14: 24, 2015.

22. Nan P, Wang T, Li C, Li H, Wang J, Zhang J, Dou N, Zhan Q, Wang H and Qian H: MTA1 promotes tumorigenesis and development of esophageal squamous cell carcinoma via activating the MEK/ERK/p90RSK signaling pathway. Carcinogenesis 41: 1263-1272, 2020 .

23. Hannafon BN, Gin AL, Xu YF, Bruns M, Calloway CL and Ding WQ: Metastasis-associated protein 1 (MTA1) is transferred by exosomes and contributes to the regulation of hypoxia and estrogen signaling in breast cancer cells. Cell Commun Signal 17: $13,2019$. 
24. Deng L, Tang J, Yang H, Cheng C, Lu S, Jiang R and Sun B: MTA1 modulated by miR-30e contributes to epithelial-to-mesenchymal transition in hepatocellular carcinoma through an ErbB2-dependent pathway. Oncogene 36: 3976-3985, 2017.

25. Liu J, Xu D, Wang H, Zhang Y, Chang Y, Zhang J, Wang J, Li C, Liu H, Zhao M, et al: The subcellular distribution and function of MTA1 in cancer differentiation. Oncotarget 5: 5153-5164, 2014.

26. Xue H, Wang H, Liu J, Liu H, Li C, Han L, Lin C, Zhan Q, Zhao Z and Qian H: MTA1 downregulation inhibits malignant potential in a small cell lung cancer cell line. Oncol Rep 33: 885-892, 2015.

27. Dhar S, Kumar A, Gomez CR, Akhtar I, Hancock JC, Lage JM, Pound CR and Levenson AS: MTA1-activated Epi-microRNA-22 regulates E-cadherin and prostate cancer invasiveness. FEBS Lett 591: 924-933, 2017.

28. Guo N, Shen G, Zhang Y, Moustafa AA, Ge D and You Z: Interleukin-17 promotes migration and invasion of human cancer cells through upregulation of MTA1 expression. Front Oncol 9: $546,2019$.

29. Yoo YG, Kong G and Lee MO: Metastasis-associated protein 1 enhances stability of hypoxia-inducible factor-1alpha protein by recruiting histone deacetylase 1. EMBO J 25: 1231-1241, 2006.

30. Weng W, Yin J,Zhang Y, Qiu J and Wang X: Metastasis-associated protein 1 promotes tumor invasion by downregulation of E-cadherin. Int J Oncol 44: 812-818, 2014.

31. Song Q, Wang B, Liu M, Ren Z, Fu Y, Zhang P and Yang M: MTA1 promotes the invasion and migration of oral squamous carcinoma by inducing epithelial-mesenchymal transition via the hedgehog signaling pathway. Exp Cell Res 382: 111450, 2019.
32. Edge S, Byrd DR and Compton CC: AJCC Cancer Staging Manual (7th edition). Springer International Publishing: American Joint Commission on Cancer, 2009.

33. Zhou N, Wang H, Liu H, Xue H, Lin F, Meng X, Liang A, Zhao Z, Liu Y and Qian H: MTA1-upregulated EpCAM is associated with metastatic behaviors and poor prognosis in lung cancer. J Exp Clin Cancer Res 34: 157, 2015.

34. Matsubara T, Toyokawa G, Takada K, Kinoshita F, Kozuma Y, Akamine T, Shimokawa M, Haro A, Osoegawa A, Tagawa T and Mori M: The association and prognostic impact of enhancer of zeste homologue 2 expression and epithelial-mesenchymal transition in resected lung adenocarcinoma. PLoS One 14: e0215103, 2019

35. Toh Y and Nicolson GL: Properties and clinical relevance of MTA protein in human cancer. Cancer Metastasis Rev 33: 891-900, 2014.

36. Wang Q, Wang F, Lv J, Xin J, Xie L, Zhu W, Tang Y, Li Y, Zhao X, Wang Y, et al: Interactive online consensus survival tool for esophageal squamous cell carcinoma prognosis analysis. Oncol Lett 18: 1199-1206, 2019.

37. Li L, Liu J, Xue H, Li C, Liu Q, Zhou Y, Wang T, Wang H, Qian $\mathrm{H}$ and Wen T: A TGF- $\beta$-MTA1-SOX4-EZH2 signaling axis drives epithelial-mesenchymal transition in tumor metastasis. Oncogene 39: 2125-2139, 2020.

(c) (i) () This work is licensed under a Creative Commons International (CC BY-NC-ND 4.0) License. 